\title{
Avaliação de frutos de goiabeira-serrana cultivados no meio oeste de Santa Catarina, Brasil
}

\author{
Fernando David Sánchez-Mora ${ }^{1 *}$ \\ Luciano Saifert ${ }^{2}$ \\ Vanessa Samara Petry ${ }^{2}$ \\ Lido José Borsuk ${ }^{2}$ \\ Juan Manuel Otalora Villamil ${ }^{2}$ \\ Rubens Onofre Nodari ${ }^{2}$ \\ ${ }^{1}$ Universidad Técnica de Manabí, Facultad de Ingeniería Agronómica \\ Campus Experimental La Teodomira, Lodana, Santa Ana, EC13132, Ecuador \\ ${ }^{2}$ Universidade Federal de Santa Catarina, Centro de Ciências Agrárias \\ Programa de Pós-Graduação em Recursos Genéticos Vegetais \\ Rodovia Admar Gonzaga, 1346, Itacorubi, CEP 88.034-001, Florianópolis - SC, Brasil \\ * Autor para correspondência \\ sanchezmorafd@gmail.com \\ fdsanchez@utm.edu.ec
}

Submetido em 28/11/2019

Aceito para publicação em $26 / 03 / 2020$

\section{Resumo}

O objetivo do presente estudo foi determinar o comportamento das características físico-químicas dos frutos de sete genótipos de goiabeira-serrana cultivados em dois locais em Santa Catarina. A avaliação foi realizada nas safras 2011/12, 2014/15 e 2015/16 nos genótipos 85, 101, 223, 458 e 509, na localidade de Fraiburgo (comunidade Papuã), e nas safras 2014/15 e 2015/16, nos genótipos 28.12 e 527, na localidade de Água Doce (comunidade Vista Alegre). Foram avaliados diâmetro $(\mathrm{cm})$, comprimento $(\mathrm{cm})$ e peso do fruto $(\mathrm{g})$, peso de casca e polpa (g), espessura de casca $(\mathrm{mm})$ e sólidos solúveis totais (grau Brix). Foram realizadas análise de variância, comparação entre médias pelo teste Tukey $(p<0,05)$ e correlações de Pearson $(p<0,05)$ entre as características dos frutos. Em Água Doce, o genótipo 527 registrou o maior diâmetro e peso do fruto $(88,7 \mathrm{~g})$. Em Fraiburgo, destacou-se o genótipo 85, que registrou o maior peso de fruto $(94,9 \mathrm{~g})$ e 509 com maior rendimento de polpa $(37,5 \%)$. Foi constatado que nem todos os genótipos com maior peso do fruto foram os que obtiveram maior rendimento de polpa, confirmando que as variáveis espessura da casca e tamanho do fruto estão diretamente relacionadas com o rendimento. A variação nos valores de sólidos solúveis totais foi afetada pela influência do ambiente e do genótipo.

Palavras-chave: Acca sellowiana; Feijoa; Qualidade de frutos

\section{Abstract}

Evaluation of feijoa fruits from two experimental orchards in the middle west of Santa Catarina, Brazil. The aim of the present study was to determine the behavior of physicochemical characteristics of feijoa 
fruits of seven genotypes grown in two locations of Santa Catarina. The evaluation was made using the following: (i) 2011/12, 2014/15 and 2015/16 harvests of genotypes 85, 101, 223, 458 and 509, cultivated in Fraiburgo (Papuã community); and (ii) 2014/15 and 2015/16 harvests of genotypes 28.12 and 527, in Água Doce (Vista Alegre community). Diameter $(\mathrm{cm})$, length $(\mathrm{cm})$ and weight $(\mathrm{g})$ of the fruit, peel $(\mathrm{g})$ and pulp $(\mathrm{g})$ weight, peel thickness $(\mathrm{mm})$, and total soluble solids (Brix grade) were evaluated. An analysis of variance, comparisons between means using Tukey's test $(p<0.05)$, and Pearson correlation coefficients $(p<0.05)$ were computed for the variables of feijoa fruits. In Água Doce, genotype 527 had the largest diameter and weight of the fruit (88.7 g). In Fraiburgo, genotype 85 had the highest fruit weight $(94.9 \mathrm{~g})$ and genotype 509 had the highest pulp yield (37.5\%). Not all genotypes with higher fruit weight exhibited higher pulp yield, confirming that the skin thickness and fruit size variables are directly related to yield. The variation in total soluble solids values was due to the environment and genotype effects.

Key words: Acca sellowiana; Feijoa; Fruit quality

\section{Introdução}

O cultivo comercial da goiabeira-serrana $[A c c a$ sellowiana (O. Berg.) Burret], fruteira nativa do Sul do Brasil e do Uruguai, ainda é incipiente decorrente tanto de tentativas dispersas de caráter experimental quanto de iniciativas dos próprios agricultores do Sul do Brasil. Mesmo assim, a goiabeira-serrana é uma cultura que vem crescendo de interesse por parte dos consumidores e também dos produtores, devido ao sabor único da fruta e à necessidade destes de obter alternativas de renda, respectivamente. Em 2012 havia o registro de pelo menos 15 agricultores que cultivavam 11 ha, os quais produziam 86,8 toneladas/ano de frutos da goiabeiraserrana no estado de Santa Catarina (CEPA, 2013). Produtores consultados informaram que nos últimos anos vendem frutos localmente e também entregaram parte da produção para mercados de Lages e São Joaquim, em SC, e Vacaria, no RS.

Estudos de mercado, desenvolvidos em dois centros comerciais do estado de Santa Catarina (Florianópolis e Blumenau), demonstraram a existência de um mercado promissor, sendo que o valor médio de comercialização dos frutos foi de aproximadamente R \$5,00/ $\mathrm{kg}$ em 2004 (BARNI et al., 2004), tendo variado o preço nos últimos anos em diferentes cidades do sul do país, de $\mathrm{R} \$ 4,00$ a R 10,00/kg, dependendo do local e tamanho dos frutos.

O fruto da goiabeira-serrana, classificado como pseudofruto do tipo pomo (MATTOS, 1986), apresenta um comportamento climatérico (AMARANTE et al., 2008) e uma variabilidade ampla, pesando entre 20 e 200 g (NODARI et al., 1997; DEGENHARDT et al.,
2003; DUCROQUET et al., 2008; MARTÍNEZ-VEGA et al., 2008). As propriedades farmacológicas existentes nos frutos têm sido mostradas por vários grupos de pesquisa. Segundo Belous et al. (2014), os frutos possuem um elevado valor biológico devido à presença de $\beta$-caroteno, vitamina $C$, agentes $\mathrm{R}$-active (catequinas e leuco-antocianinas) e um complexo de valiosos micro e macro elementos. O complexo de ligações fenólicas (catequinas e leuco-antocianinas) causa a atividade da vitamina $\mathrm{P}$ e dá ao fruto um sabor adstringente. Foram reportadas atividades antibactericidas e antioxidantes (BASILE et al., 1997; VUOTTO et al., 2000), antidepressiva significativa (MAHMOUDI et al., 2015), antitumorais (BONTEMPO et al., 2007), contribuições para tratamento e prevenção do diabetes tipo 2 e artrite reumatoide (patente US 20150157678; FOO; WARSON, 2015).

O programa de melhoramento da goiabeiraserrana, realizado em conjunto pela EPAGRI, Estação Experimental São Joaquim, e pela UFSC, estabeleceu critérios para a seleção dos melhores acessos em relação às características dos frutos, entre elas: regularidade da produção; peso $>70 \mathrm{~g}$; rendimento em polpa $>30 \%$ ou com casca comestível; sabor agradável; tolerância à antracnose; período de maturação variável e formato do fruto. Assim, os acessos selecionados devem cumprir com algumas ou todas as características de interesse. A estes, outros atributos podem ser adicionados, como produtividade e estabilidade; frutos grandes e uniformes; bom formato e aparência dos frutos. Como resultado de cruzamentos anteriores e após duas décadas de trabalho foram lançadas as primeiras variedades comerciais de 
goiabeira-serrana (DUCROQUET et al., 2007; 2008). Dessa forma, os resultados do presente estudo podem ser utilizados tanto para recomendação de cultivo como para auxiliar na escolha de parentais para o próximo ciclo de cruzamentos.

Experiências no programa de melhoramento genético participativo da goiabeira-serrana indicaram que os principais critérios escolhidos pelos agricultores foram fruto de maior tamanho, maior rendimento de polpa e sabor (DONAZZOLO, 2012; SANTOS et al., 2017). Uma estratégia de promoção da goiabeiraserrana junto a produtores da região de São Joaquim foi a alocação de mudas em propriedades agrícolas com o intuito de avaliar a adaptação dos materiais, ao mesmo tempo em que os produtores se familiarizavam com seu manejo (SANTOS et al., 2018).

Visando estudar o comportamento da goiabeiraserrana cultivada em vários locais com altitudes diferentes em Santa Catarina, foram estabelecidos no início do século pomares experimentais nas localidades de Água Doce e Fraiburgo. Assim, no presente estudo o objetivo foi avaliar as características físico-químicas dos frutos de genótipos do Banco Ativo de Germoplasma (BAG) de goiabeira-serrana mantidos pela EPAGRI de São Joaquim.

\section{Material e Métodos}

Os sete genótipos utilizados são provenientes do Banco Ativo de Germoplasma (BAG) da feijoa mantido pela EPAGRI, Estação Experimental São Joaquim, e foram selecionados para avaliações em outros locais, por se destacarem em relação a características físicoquímicas do fruto em avaliações realizadas no BAG.

As avaliações das características dos frutos de goiabeira-serrana foram realizadas em dois experimentos: o primeiro experimento foi implantado no ano 2002, no município de Fraiburgo, Meio Oeste Catarinense, na comunidade Papuã, propriedade do Sr. Leodir Francescatto (latitude $27^{\circ} 00^{\prime} \mathrm{S}$, longitude $51^{\circ} 00^{\prime} \mathrm{W}$ e altitude de $1.014 \mathrm{~m})$. Foram estabelecidos os genótipos $85,101,223,458$ e 509 , em delineamento experimental de blocos ao acaso com seis tratamentos correspondentes a cada genótipo, com três blocos e três repetições de cada tratamento. O espaçamento foi de $6,0 \mathrm{~m}$ x 4,0 m numa área de $1.296 \mathrm{~m}^{2}$, totalizando 54 plantas.

Os principais tratos culturais foram: poda de inverno, raleio dos frutos, adubação química fornecida pela N-ureia e Cloreto de Potássio (KCl) na dose de 400 $\mathrm{g}$ por planta. $\mathrm{O}$ controle de ervas daninha foi realizado manualmente no contorno da planta combinado de aplicações químicas de glifosato (Glyphosate ${ }^{\circledR}$ ) nas entrelinhas. As avaliações dos frutos foram realizadas durante os ciclos de 2011/12, 2014/15 e 2015/16.

O segundo experimento foi estabelecido no ano 2005, no município de Água Doce, Meio Oeste Catarinense, na comunidade Vista Alegre, propriedade do Sr. Vercedino Freitas (latitude $26^{\circ} 52^{\prime}$ S, longitude $51^{\circ} 32^{\prime} \mathrm{W}$ e altitude de $1.250 \mathrm{~m}$ ). Foram estabelecidos os genótipos 28.12 e 527 em delineamento em blocos ao acaso, e multiplicados em sete porta-enxertos diferentes $(101 \times 458,509 \times 85,509 \times 101,509 \times 458$, 53B7 x 101, 53B 7 x 458 e 85 x 101), com três blocos e três repetições de cada tratamento. No total 66 plantas foram estabelecidas. O espaçamento utilizado foi de 5 $\mathrm{m} \times 3,5 \mathrm{~m}$.

Os principais tratos culturais foram a poda de inverno e o raleio dos frutos. Quanto à adubação foram utilizados $5 \mathrm{~kg}$ de cama de aviário e $400 \mathrm{~g}$ por planta de uma mistura composta de Cloreto de Potássio (KCl), Superfosfato Triplo (SFT) e N-ureia. Após a adubação, realizou-se a aplicação de herbicida glifosato (Glyphosate ${ }^{\circledR}$ ) nas entrelinhas para o controle de ervasdaninhas. As avaliações dos frutos foram realizadas durante os ciclos de 2014/15 e 2015/16.

De cada genótipo avaliado foi coletada uma amostra de 10 frutos (DEGENHARDT et al., 2002; SÁNCHEZ-MORA et al., 2019), no ponto de colheita comercial (quando se desprendem facilmente da planta). As amostras foram transportadas para o Laboratório de Fisiologia do Desenvolvimento e Genética Vegetal (LFDGV) da UFSC, onde foram realizadas as avaliações. Foram avaliados o diâmetro do fruto $(\mathrm{cm})$, medido em dois lados da parte equatorial do fruto; comprimento do fruto $(\mathrm{cm})$ e espessura da casca $(\mathrm{mm})$ com o auxílio de um paquímetro digital. 
O peso total do fruto $(\mathrm{g})$, peso da casca $(\mathrm{g})$ e peso da polpa (g) foram estimados com o auxílio de balança de precisão (Shimadzu BL 3200H). Posteriormente, o rendimento da polpa em percentagem (\%) foi estimado pela fração do peso da polpa e o peso total do fruto. Os sólidos solúveis totais ( $\mathrm{SST},{ }^{\circ} \mathrm{Brix}$ ) foram medidos com o auxílio de um refratômetro portátil digital 0 a $85 \%$ Brix (HANNA instruments, modelo HI 96801).

Os dados obtidos foram submetidos à análise de variância (ANOVA) e, quando verificada a significância, procedeu-se à comparação de médias, pelo teste de Tukey, a 5\% de probabilidade, sendo considerados os genótipos e os anos como fatores. Análises de correlação de Pearson também foram realizadas. Empregou-se o software livre R (R DEVELOPMENT CORE TEAM, 2017).

\section{Resultados}

A análise de variância mostrou significância estatística para diferenças nas características avaliadas nos frutos de goiabeira-serrana, entre genótipos e entre anos e para a interação genótipos $\mathrm{x}$ ano, nos experimentos conduzidos em Água Doce e Fraiburgo. No experimento de Água Doce, foi verificado que o fator porta-enxerto não foi significativo, razão pela qual foi desconsiderado como causa de variação.

As diferenças encontradas na característica diâmetro do fruto foram estatisticamente significativas $(\mathrm{p}<0,01)$ em genótipos e anos (Tabela 1) na localidade de Água Doce; enquanto em Fraiburgo encontrou-se significância estatística $(\mathrm{p}<0,01)$ entre genótipos e na interação genótipos x anos. Em Água Doce o genótipo

TABELA 1: Médias de diâmetro do fruto (DIA), comprimento do fruto (COM); peso do fruto (PESO); rendimento de polpa (REND); espessura de casca (ESP) e Sólidos Solúveis Totais (SST) de goiabeira-serrana avaliados nas localidades de Água Doce (safras 2014/15 e 2015/16) e Fraiburgo (safras 2011/12, 2014/15 e 2015/16), SC, Brasil.

\begin{tabular}{|c|c|c|c|c|c|c|}
\hline ÁGUA DOCE ${ }^{1}$ & $\begin{array}{l}\text { DIA } \\
\text { (cm) }\end{array}$ & $\begin{array}{c}\text { COM } \\
(\mathrm{cm})\end{array}$ & $\begin{array}{c}\text { PESO } \\
\text { (g) }\end{array}$ & $\begin{array}{c}\text { REND } \\
(\%)\end{array}$ & $\begin{array}{l}\text { ESP } \\
(\mathrm{cm})\end{array}$ & $\begin{array}{c}\text { SST } \\
\left({ }^{\circ} \text { Brix }\right)\end{array}$ \\
\hline GENÓTIPOS (p para o fator A) & $* *$ & $* *$ & $* *$ & NS & $*$ & $* *$ \\
\hline 28.12 & $4,7 \mathrm{~b}$ & $6,6 \mathrm{a}$ & $77,9 \mathrm{~b}$ & 29,5 & $0,7 \mathrm{~b}$ & $12,5 \mathrm{a}$ \\
\hline 527 & $5,2 \mathrm{a}$ & $6,1 \mathrm{~b}$ & 88,7 a & 29,2 & $0,8 \mathrm{a}$ & $11,1 \mathrm{~b}$ \\
\hline ANOS (p para o fator B) & $* *$ & NS & $* *$ & $* *$ & $* *$ & $* *$ \\
\hline 2015 & $4,7 \mathrm{~b}$ & 6,4 & $76,6 \mathrm{~b}$ & $33,6 \mathrm{a}$ & $0,7 \mathrm{~b}$ & $13,5 \mathrm{a}$ \\
\hline 2016 & $5,0 \mathrm{a}$ & 6,4 & $87,1 \mathrm{a}$ & $26,5 \mathrm{~b}$ & $0,8 \mathrm{a}$ & $10,7 \mathrm{~b}$ \\
\hline Interação A x B & NS & $* *$ & * & $* *$ & NS & NS \\
\hline CV $(\%)$ & 6,6 & 6,7 & 15,6 & 11,0 & 13,2 & 10,9 \\
\hline \multicolumn{7}{|l|}{ FRAIBURGO $^{2}$} \\
\hline GENÓTIPOS (p para o fator A) & $* *$ & $* *$ & $* *$ & $* *$ & $* *$ & $* *$ \\
\hline 85 & $5,1 \mathrm{a}$ & $6,7 \mathrm{a}$ & 94,9 a & $33,4 \mathrm{c}$ & $0,7 \mathrm{a}$ & $11,9 \mathrm{~b}$ \\
\hline 101 & $4,8 \mathrm{~b}$ & $6,0 \mathrm{~b}$ & $68,3 \mathrm{~b}$ & $32,4 \mathrm{~d}$ & $0,6 \mathrm{c}$ & $13,3 \mathrm{a}$ \\
\hline 223 & $4,7 \mathrm{~b}$ & $5,7 \mathrm{c}$ & $69,5 \mathrm{~b}$ & $33,2 \mathrm{c}$ & $0,7 \mathrm{~b}$ & $11,5 \mathrm{c}$ \\
\hline 458 & $3,8 \mathrm{~d}$ & $5,2 \mathrm{~d}$ & $42,0 \mathrm{~d}$ & $35,0 \mathrm{~b}$ & $0,5 \mathrm{e}$ & $11,5 \mathrm{c}$ \\
\hline 509 & $4,3 \mathrm{c}$ & $5,0 \mathrm{e}$ & $51,5 \mathrm{c}$ & $37,5 \mathrm{a}$ & $0,5 \mathrm{~d}$ & $11,8 \mathrm{~b}$ \\
\hline ANOS (p para o fator B) & NS & NS & NS & $* *$ & $* *$ & $* *$ \\
\hline 2012 & 4,5 & 5,7 & 62,2 & $36,7 \mathrm{a}$ & $0,6 \mathrm{~b}$ & $10,8 \mathrm{c}$ \\
\hline 2015 & 4,7 & 5,6 & 68,5 & $33,6 \mathrm{~b}$ & $0,5 \mathrm{c}$ & $13,2 \mathrm{a}$ \\
\hline 2016 & 4,6 & 5,8 & 69,5 & $32,4 \mathrm{c}$ & $0,7 \mathrm{a}$ & $12,3 \mathrm{~b}$ \\
\hline INTERAÇÃO A x B & $* *$ & $* *$ & $* *$ & $*$ & $* *$ & $* *$ \\
\hline $\mathrm{CV}(\%)$ & 7,1 & 8,5 & 22,4 & 10,7 & 13,6 & 7,6 \\
\hline
\end{tabular}

${ }^{1}$ Médias seguidas de mesma letra não diferem entre si, pelo teste F. ${ }^{2}$ Médias seguidas de mesma letra não diferem entre si, pelo teste de Tukey. NS = não significativo, * $\mathrm{e}^{* *}$ significativo a 5 e $1 \%$ de probabilidade, respectivamente. 
527 produziu frutos com o maior valor do diâmetro (5,2 cm), em comparação com 28.12 (4,7 cm). Em Fraiburgo, o genótipo 85 registrou o maior diâmetro do fruto, com $5,1 \mathrm{~cm}$, enquanto o genótipo 458 produziu frutos menores $(3,8 \mathrm{~cm})$. Na característica comprimento do fruto encontraram-se diferenças significativas $(p<0,01)$ em genótipos e na interação genótipos $\mathrm{x}$ anos (Tabela 1) nas duas localidades. Em Água Doce os frutos do genótipo 28.12 foram os mais compridos $(6,6 \mathrm{~cm})$, já em Fraiburgo foi o genótipo 85 que produziu os frutos mais compridos $(6,7 \mathrm{~cm})$.

As características diâmetro e peso do fruto apresentaram as mesmas significâncias estatísticas nos genótipos nas duas localidades (Tabela 1). Com relação ao peso do fruto, houve diferenças $(\mathrm{p}<0,01)$ para genótipos, anos e a interação genótipos $\mathrm{x}$ anos em Água Doce; em Fraiburgo foram verificadas diferenças $(p<0,01)$ entre genótipos e para a interação genótipos $\mathrm{x}$ anos (Tabela 1). Em Água Doce o peso do fruto variou de 77,9 g (28.12) a 88,7 g (527); enquanto em Fraiburgo verificou-se que o genótipo 85 registrou o maior peso $(94,9 \mathrm{~g})$, e o menor peso foi dos frutos do genótipo 458 $(42,0 \mathrm{~g})$.

Os genótipos em Água Doce apresentaram valores similares para o rendimento de polpa (29\%), enquanto em Fraiburgo destacou-se o genótipo 509 (37,5\%), sendo que o menor rendimento de polpa (32,4\%) foi revelado pelo genótipo 101. Quanto aos sólidos solúveis totais, em Água Doce, foram verificados valores de 11,1 e 12,5\% ${ }^{\circ}$ Brix, para os genótipos 527 e 28.12, respectivamente. Nos frutos em Fraiburgo constataram-se valores médios de $11,5^{\circ}$ Brix, nos genótipos 223 e 458 e o maior valor (13, $3^{\circ}$ Brix) no genótipo 101 (Tabela 1). Em Fraiburgo, os frutos do genótipo 101 produziram os maiores valores de ${ }^{\circ}$ Brix nos três anos de avaliação. Os genótipos 85, 223 e 458 incrementaram seu ${ }^{\circ}$ Brix com o passar do tempo, enquanto os valores SST reduziram no genótipo 509 no período avaliado (Figura 1).

FIGURA 1: Interações significativas entre os fatores genótipos e anos nas características dos frutos. Localidade de Fraiburgo, SC: Diâmetro (A), Comprimento (B), Peso do fruto (C), Rendimento de polpa (D), Espessura de casca (E) e Sólidos solúveis totais (F); e na Localidade Água Doce, SC: Comprimento (G), Peso (H) e Rendimento de polpa (I).

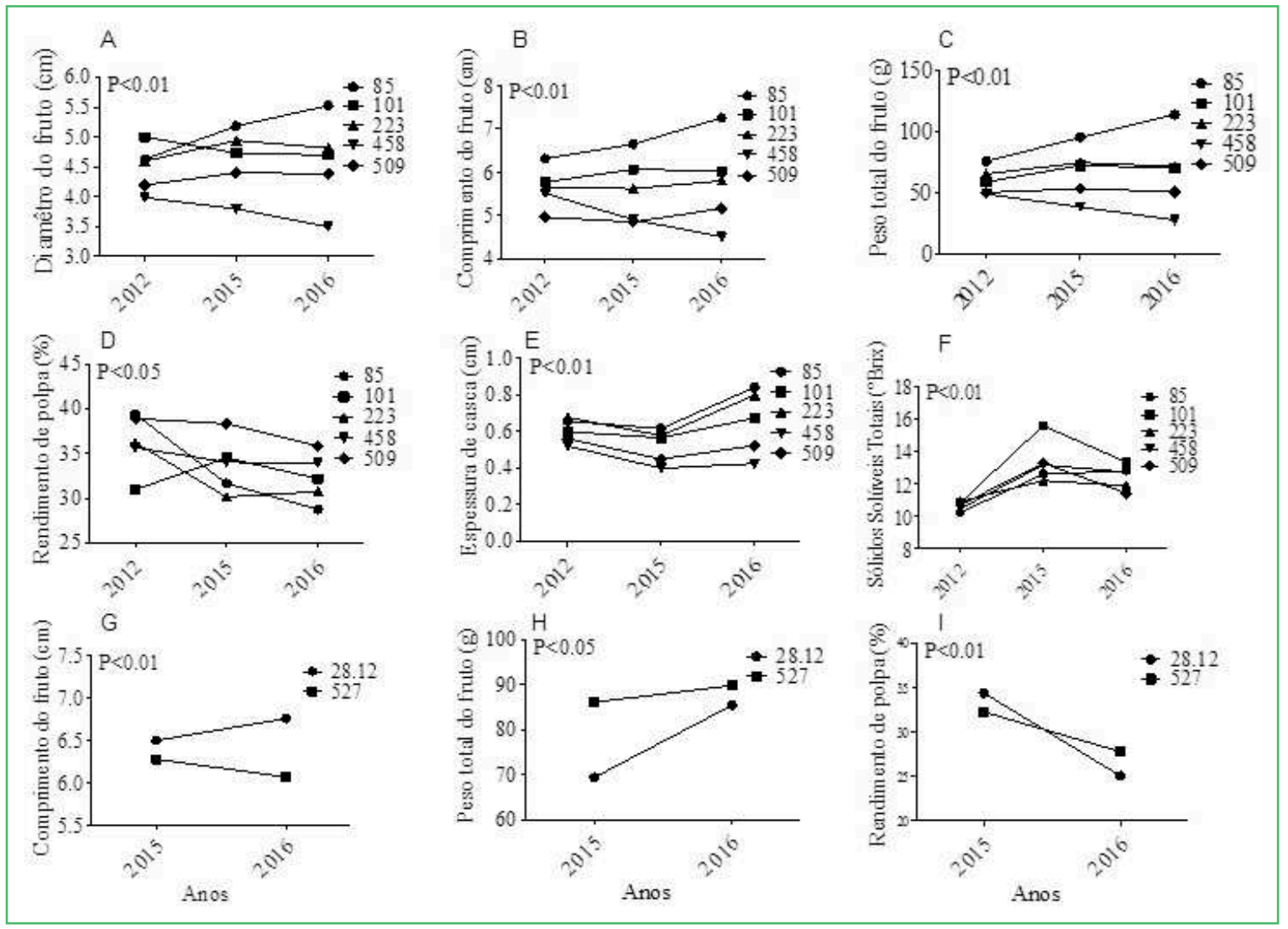


No decorrer dos anos, constatou-se em Água Doce que o genótipo 28.12 aumentou o peso e o comprimento do fruto, mas houve redução no rendimento de polpa. No entanto, os frutos do genótipo 527 diminuíram em diâmetro e rendimento de polpa, mas mantiveram o peso de fruto no período de avaliação (Figura 1). Em Fraiburgo os genótipos apresentam um comportamento similar em relação às características dos frutos. Assim, os frutos dos genótipos 85 e 509, no decorrer dos anos de avaliação, aumentaram em diâmetro, comprimento, peso e em espessura de casca do fruto, mas houve redução do rendimento de polpa. Por outro lado, os frutos do genótipo 458 reduziram em diâmetro, comprimento, peso do fruto e rendimento de polpa, mas aumentaram a espessura da casca. O genótipo 101 manteve os valores de diâmetro, comprimento e peso de fruto, mas apresentou menor rendimento de polpa e maior espessura de casca (Figura 1).

Na Figura 2, observa-se que existem coeficientes de correlação altamente significativos entre o peso do fruto e as características de diâmetro (Fraiburgo, $\mathrm{r}=$ 0,92-0,95; Água Doce, $\mathrm{r}=0,80-0,85)$, comprimento (Fraiburgo, r = 0,85-0,90; Água Doce, $r=0,65-0,66$ ) e espessura de casca (Fraiburgo, $r=0,61-0,71$; Água Doce, $r=0,50)$. Assim, também foi constatado um coeficiente de correlação, importante para o melhoramento, entre o rendimento de polpa (\%) e a espessura de casca (Fraiburgo, $\mathrm{r}=-0,40$ a -0,55; Água Doce, $\mathrm{r}=-0,38 \mathrm{a}$ $-0,46)$. O diâmetro do fruto também está relacionado com a espessura de casca (Fraiburgo, $r=0,62$ a 0,73; Água Doce, $r=0,45$ ). Os sólidos solúveis totais (SST) têm uma ligeira correlação com o rendimento de polpa $(r=-0,13)$.

\section{Discussão}

A não significância do fator porta-enxerto no experimento implantado em Água Doce indica que esse fator não tem influência nas características físicoquímicas do fruto de goiabeira-serrana. Esse resultado concorda com resultados de estudos anteriores que demonstraram que os mesmos porta-enxertos não afetaram o desenvolvimento vegetativo e a qualidade de frutos de goiabeira-serrana (SANTOS et al., 2013).
Os valores de diâmetro e comprimento do fruto representam o desenvolvimento normal da goiabeiraserrana nas localidades avaliadas e são similares aos valores de variedades comerciais. No presente trabalho os intervalos encontrados para diâmetro e comprimento do fruto em Fraiburgo $(3,8-5,1 \mathrm{~cm} \mathrm{e}$ $5,0-6,7 \mathrm{~cm})$ e Água Doce (4,7-5,2 cm e 6,1-6,6 cm), respectivamente, podem ser considerados de tamanho médio para a espécie. Esses valores médios são similares aos encontrados para outras variedades da mesma espécie e cultivadas em outra localidade, pois frutos da variedade colombiana "Quimba" atingiram os seguintes intervalos de diâmetro $(32,49 \pm 2,57 \mathrm{~mm}$ a 49,07 $\pm 2,45$ $\mathrm{mm})$ e de comprimento de fruto $(57,35 \pm 3,29 \mathrm{~mm}$ a $76,19 \pm 3,25 \mathrm{~mm}$ ) (PARRA-CORONADO et al., 2015). Entretanto, menores intervalos de diâmetro (2,3 a 3,9 $\mathrm{cm})$ e de comprimento de fruto $(2,8$ a $6,0 \mathrm{~cm})$ foram registrados em uma população de 300 plantas na Turquia (BEYHAN; EYDURAN, 2011).

As características de diâmetro e peso do fruto encontraram-se estreitamente relacionadas nos genótipos avaliados nas duas localidades. Esse resultado mostra a elevada correlação dessas duas variáveis. Segundo Donazzolo et al. (2017), o peso total do fruto, seguido do diâmetro, tem efeito direto e é uma das principais caracteristicas associadas ao peso de polpa, fator de grande importância comercial.

Os maiores pesos do fruto registrados nas duas localidades são similares aos encontrados nas variedades brasileiras de goiabeira-serrana, sendo que em média a variedade Alcântara produz frutos de $80 \mathrm{~g}$, Nonante de $90 \mathrm{~g}$ e de $150 \mathrm{~g}$ nas variedades Mattos e Helena (DUCROQUET et al., 2007; 2008). $\mathrm{Na}$ variedade colombiana Quimba, o peso do fruto varia de 21,1 a 98,9 g (MARTÍNEZ-VEGA et al., 2008; PARRA-CORONADO et al., 2015), enquanto em variedades neozelandesas a média do peso do fruto é $73 \mathrm{~g}$, variando de 51,8 (Variedade Smith) a 104,8 (Mammoth) (PASQUARIELLO et al., 2015). Dessa maneira, os genótipos avaliados nas localidades de Água Doce e Fraiburgo apresentam características dos frutos adequadas para a comercialização ou como parentais em novos cruzamentos. 
FIGURA 2: Coeficientes de correlação entre as características do fruto: Diâmetro (DIA), Comprimento (COM), Peso do fruto (PESO), Rendimento de polpa (REND), Espessura de casca (ESP) e Sólidos solúveis totais (SST) durante as safras 2011/12, 2014/15 e 2015/16 nas localidades de Fraiburgo e Água Doce, SC, Brasil. A intensidade da cor e o tamanho do círculo são proporcionais aos coeficientes de correlação.

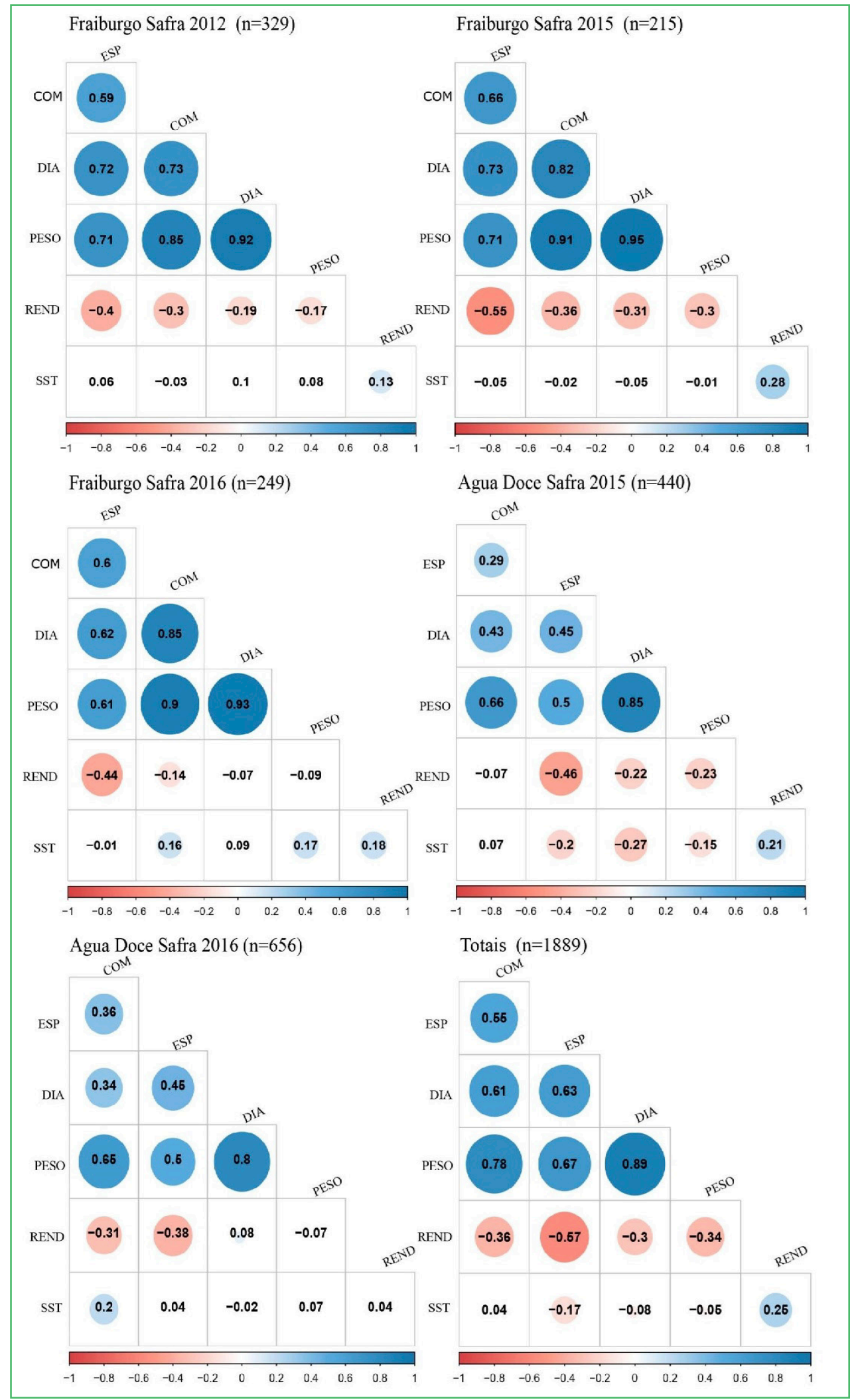


Os rendimentos de polpa dos genótipos cultivados em Água Doce e Fraiburgo foram satisfatórios e se enquadram no intervalo de valores mostrados pelas variedades brasileiras, que em média registram rendimentos de polpa variando 25 a $32 \%$ na cultivar Helena, e entre $27 \%$ e $33 \%$ nas cultivares Nonante e Mattos (DUCROQUET et al., 2007; 2008).

Em relação aos sólidos solúveis totais ( ${ }^{\circ}$ Brix), foi constatado que genótipos (ex: 101) conseguem manter valores superiores nessa característica, comparativamente aos demais genótipos testados, ao longo dos anos. Esse fato sugere que outros genótipos são mais afetados pelo ambiente, o que causa diminuição no seu conteúdo de açúcares de um ano para outro. Segundo Ducroquet et al. (2007; 2008), os sólidos solúveis totais das variedades de goiabeiraserrana são considerados médios se comparados aos dos outros acessos (por exemplo, L203C212 - 18,3\% SST no ano 2006/07) e variam dependendo do ano. Os autores do referido estudo constataram que o teor de sólidos solúveis em média varia de 11 a 13\% em Alcântara, de 9 a 14\% em Helena, entre 10 e 13\% em Mattos e Nonante. Na variedade Quimba (Colômbia) os valores de SST oscilaram entre 11,7 e $13,4^{\circ}$ Brix na localidade de Tenjo (PARRA-CORONADO et al., 2015). Na Nova Zelândia foram reportados frutos com média de 10,5 de SST na variedade Mammoth (HARMAN, 1987).

Esses resultados, sobre a correlação das características dos frutos e sua variação de uma safra com a seguinte, demonstram a complexidade para os melhoristas, pois em anos diferentes a associação entre características não se mantém nas mesmas proporções. Além disso, indicam que estudos de avaliação final de genótipos para fins de recomendação de cultivo devem ser realizados durante pelo menos três anos de avaliação nos locais onde se pretende incentivar o cultivo.

As correlações significativas das características do fruto podem ser confirmadas com a análise do total de frutos das duas localidades, mostrando também que o rendimento de polpa é correlacionado negativamente para as características de comprimento, diâmetro e peso do fruto. Segundo Degenhardt et al. (2003), o aumento no rendimento está relacionado, ainda que fracamente, a uma diminuição no conteúdo de SST, principalmente quando o peso médio dos frutos é maior.

A correlação entre o rendimento de polpa e a espessura de casca já foi avaliada anteriormente em outros estudos (DEGENHARDT et al., 2003; DONAZZOLO et al., 2017). No estudo em que foram utilizados frutos de 229 acessos do BAG, as características peso total de fruto, diâmetro do fruto e espessura da casca estão fortemente relacionadas ao rendimento da polpa (SÁNCHEZ-MORA et al., 2019). Assim, nem todos os acessos com maior peso do fruto produzirão o maior rendimento de polpa, pois quanto maior o peso de fruto, também poderá ser maior o peso da casca, o comprimento e o diâmetro do fruto (DEGENHARDT et al., 2003), a depender das condições climáticas e do manejo. Consequentemente, não foi surpresa que nem todos os genótipos com maior peso do fruto foram os que apresentaram maior rendimento de polpa. Por fim, confirmam-se os resultados anteriores de que as variáveis espessura de casca e tamanho do fruto possam estar diretamente relacionadas com o rendimento de polpa.

\section{Agradecimentos}

Aos Senhores Leodir Francescatto e Vercedino Freitas por permitirem que o trabalho fosse feito em suas propriedades; à Fundação de Amparo à Pesquisa do Estado de Santa Catarina (FAPESC; Proj. 5288/2011-4 and 2780/2012-4), ao Conselho Nacional de Desenvolvimento Científico e Tecnológico (CNPq) (Proj. 478270/2012-7) pelos recursos financeiros e pelas bolsas de estudos para RON e LS, à Coordenação de Aperfeiçoamento de Pessoal de Nível Superior - Brasil (CAPES) - Código de Financiamento 001, e à Secretaria Nacional de Educación Superior, Ciencia, Tecnología e Innovación - Ecuador (SENESCYT).

\section{Referências}

AMARANTE, C. D.; STEFFENS, C. A.; DUCROQUET, J. P. H. J.; SASSO, A. Qualidade de goiaba serrana em resposta à temperatura de armazenamento e ao tratamento com 1-metilciclopropeno. Pesquisa Agropecuária Brasileira, Brasília, v. 43, n. 12, p. 16831689,2008 
BARNI, E. J.; DUCROQUET, J. P.; SILVA, M. C.; NETO, R. B.; PRESSER, R. F. Potencial de mercado para goiabeira-serrana catarinense. Florianópolis: Epagri, 2004. 48 p. Documento 212.

BASILE, A.; VUOTTO, M. L.; VIOLANTE, U.; SORBO. S.; MARTONE. G.; CASTALDO-COBIANCHI. R. Antibacterial activity in Actinidia chinensis, Feijoa sellowiana and Aberia caffra. International Journal of Antimicrobial Agents, Oxford, v. 8, n. 3, p. 199-203, 1997.

BELOUS, O.; OMAROV, M.; OMAROVA, Z. Chemical composition of fruits of a feijoa (Feijoa sellowiana) in the conditions of subtropics of Russia. Scientific Journal for Food Industry, Nitrianske Hrnčiarovce, v. 8, n. 1, p. 119-123, 2014.

BEYHAN, O.; EYDURAN, S. P. Determination of promising native Feijoa (Feijoa sellowiana Berg.) genotypes from Sakarya Region in Turkey. Scientific Research and Essays, Abraka, v. 6, n. 19, p. 4104-4108, 2011.

BONTEMPO, P.; MITA, L.; MICELI, M.; DOTO, A.; NEBBIOSO, A.; DE BELLIS, F.; CONTE, M.; MINICHIELLO, A.; MANZO, F.; CARAFA, V.; BASILE, A.; RIGANO, D.; SORBO, S.; CASTALDO COBIANCHI, R.; SCHIAVONE, E. M.; FERRARA, F.; de SIMONE, M.; VIETRI, M.; CIOFFI, M.; SICA, V.; BRESCIANI, F.; de LERA, A. R.; ALTUCCI, L.; MOLINARI, A. M. Feijoa sellowiana, derived natural Flavone exerts anti-cancer action displaying HDAC inhibitory activities. International Journal of Biochemistry \& Cell Biology, London, v. 39, n. 10, p. 1902-1914, 2007.

CEPA - CENTRO DE SOCIOECONOMIA E PLANEJAMENTO AGRÍCOLA. Síntese anual da agricultura de Santa Catarina 2011-2012. Florianópolis: EPAGRI, 2013. 182 p.

DEGENHARDT, J.; DUCROQUET, J. P.; GUERRA, M. P.; NODARI, R. O. Avaliação fenotípica de características de frutos em duas famílias de meios-irmãos de goiabeira-serrana (Acca sellowiana Berg.) de um pomar comercial em São Joaquim, SC. Revista Brasileira de Fruticultura, Jaboticabal, v. 25, n. 3, p. 475479, 2003.

DEGENHARDT, J.; DUCROQUET, J. P.; REIS, M. S.; GUERRA, M. P.; NODARI, R. O. Efeito de anos e determinação do coeficiente de repetibilidade de características de frutos de goiabeira-serrana. Pesquisa Agropecuária Brasileira, Brasília, v. 37, n. 9, p. 1285 1293, 2002.

DONAZZOLO, J. Conservação pelo uso e domesticação da feijoa na serra gaúcha - RS. 2012. 312 f. Tese (Doutorado em Recursos Genéticos Vegetais) - Universidade Federal de Santa Catarina, Florianópolis. 2012.

DONAZZOLO, J.; SALLA, V. P.; SASSO, S. A. Z.; DANNER, M. A.; CITADIN, I.; NODARI, R. O. Path analysis for selection of feijoa with greater pulp weight. Ciência Rural, Santa Maria, v. 47, n. 6, e20161062, 2017.

DUCROQUeT, J. P. H. J.; NUNES, E. C.; GUERRA, M. P.; NODARI, R. O. Novas cultivares brasileiras de goiabeira-serrana: SCS 414-Mattos e SCS 415-Nonante. Agropecuária Catarinense, Florianópolis, v. 21, n. 2, p. 79-82, 2008.

DUCROQUET, J. P. H. J.; SANTOS, K. L.; ANDRADE, E. R.; BONETI, J. I.; BONIN, V.; NODARI, R. O. As primeiras cultivares brasileiras de goiabeira serrana: SCS 411 Alcântara e SCS 412 Helena. Agropecuária Catarinense, Florianópolis, v. 20, n. 2, p. 77-80, 2007.
FOO, L. Y.; WATSON, R. R. Feijoa fruit extract. US Patent. 20150157678:A1. 2015.

HARMAN, E. Feijoa fruit: growth and chemical composition during development. New Zealand Journal of Experimental Agriculture, Auckland, v. 15, n. 2, p. 209-215, 1987.

MAHMOUDI, M.; EBRAHIMZADEH, M. A.; ABDI, M.; ARIMI, Y.; FATHI, H. Antidepressant activities of Feijoa sellowiana fruit. European Review for Medical and Pharmacological Sciences, Roma, v. 19, p. 2510-2513, 2015.

MARTÍNEZ-VEGA, R. R.; FISCHER, G.; HERRERA, A.; CHAVES, B.; QUINTERO, O. C. Características físico-químicas de frutos de feijoa influenciadas por la posición en el canopi. Revista Colombiana de Ciencias Hortícolas, Bogotá, v. 2, n. 1, p. 21-32, 2008.

MATTOS, J. R. A goiabeira-serrana. Porto Alegre: Instituto de Pesquisas de Recursos Naturais Renováveis, 1986. 84 p. (Publicação IPRNR, 19).

NODARI, R. O.; GUERRA, M. P.; MELER, K. T.; DUCROQUET, J. P. Genetic variability of Feijoa sellowiana germplasm. Acta Horticulturae, Leuven, v. 452, p. 41-45, 1997.

PARRA-CORONADO, A.; FISCHER, G.; CAMACHOTAMAYO, J. H. Development and quality of pineapple guava fruit in two locations with different altitudes in Cundinamarca, Colombia. Bragantia, Campinas, v. 74, n. 3, p. 359-366, 2015.

PASQUARIELLO, M. S.; MASTROBUONI, F.; DI PATRE, D.; ZAMPELLA, L.; CAPUANO, L. R.; SCORTICHINI, M.; PETRICCIONE, M. Agronomic, nutraceutical and molecular variability of feijoa (Acca sellowiana (O. Berg) Burret) germplasm. Scientia Horticulturae, Amsterdam, v. 191, p. 1-9, 2015.

R DEVELOPMENT CORE TEAM. R: A language and environment for statistical computing. Vienna: R Foundation for Statistical Computing, 2017. Disponível em: https://www.R-project.org/.

SÁNCHEZ-MORA，F. D.; SAIFERT, L.; CIOTTA, M. N.; RIBEIRO, H. N.; PETRY, V. S.; ROJAS-MOLINA, A. M.; LOPES, M. E.; LOMBARDI, G. G.; SANTOS, K. L.; DUCROQUET, J. P. H. J.; NODARI, R. O. Characterization of phenotypic diversity of Feijoa fruits of germplasm accessions in Brazil. Agrosystems, Geosciences \& Environment, Madison, v. 2, p. 1-11, 2019.

SANTOS, K. L.; CIOTTA, M. N.; DONAZZOLO, J.; BORSUK, L. J.; NODARI, R. O. Domesticação da espécie. In: CIOTTA, M. N.; ARIOLI, C. J.; PINTO, F. A. M. F.; SANTOS, K. L.; ARAUJO, L.; PASA, M. S. (Ed.). A cultura da goiabeira-serrana. Florianópolis: Epagri, 2018. 216 p.

SANTOS, K. L.; DONAZZOLO, J.; GURIES, R. P.; PERONI, N.; NODARI, R. O. Participatory research with stimulating the breeding process for a native fruit species. Agroecology and Sustainable Food Systems, London, v. 42, n. 4, p. 432-447, 2017.

SANTOS, K. L.; KATSURAYAMA, A. Y.; DUCROQUET, J. P. H. J. Avaliação de genótipos de goiabeira-serrana para utilização como porta-enxertos de cultivares comerciais. Agropecuária Catarinense, Florianópolis, v. 26, n. 1, p. 52-55, 2013.

VUOTTO, M. L.; BASILE, A.; MOSCATIELlO, V.; SOLE, P.; CASTALDO-COBIANCHI, R.; LAGHI, E.; IELPO, M. T. L. Antimicrobial and antioxidant activities of Feijoa sellowiana fruit. International Journal of Antimicrobial Agents, Oxford, v. 13, n. 3, p. 197-201, 2000. 